\title{
Fabrication of DNA Concatemers Containing Multiple Modifications
}

\author{
Liat Katrivas ${ }^{1, *}$, and Alexander Kotlyar ${ }^{l,}$ *
}

1) Department of Biochemistry and Molecular Biology, George S. Wise Faculty of Life Sciences and the Center of Nanoscience and Nanotechnology, Tel Aviv University, Ramat Aviv, Tel Aviv 69978, Israel

${ }^{*}$ E-mail liatkatrivas@mail.tau.ac.il

Here we describe the construction of double stranded (ds) concatemers composed of short (610 bases) repeating tandem oligonucleotides units containing modified nucleotides. The synthesis of incudes: self-association of the 5'-end phosphorylated half-complementary oligonucleotides into long (hundreds of base pairs) ds nucleic acid polymers and covalent linkage of the oligonucleotides in the strand by DNA ligase (Fig. 1B). The oligonucleotides containing various modified nucleotides can be assembled into long (hundreds of nanometers) ds concatamers. One of these nucleotides is amino-modified thymine in which the amino group is linked to 5-position of the pyrimidine ring via two or six carbon atom spacer arms. The amine group can be conjugated to amine reactive derivatives of various organic dyes and nanostructures. However, since the presence of modified bases should affect the base stacking in DNA in this research we have been mostly focused on attached of functional groups to the nucleic acid's backbone. This was done through conjugation of the iodoacetamide derivatives of molecules of interest with a phosphothioate group (groups) of
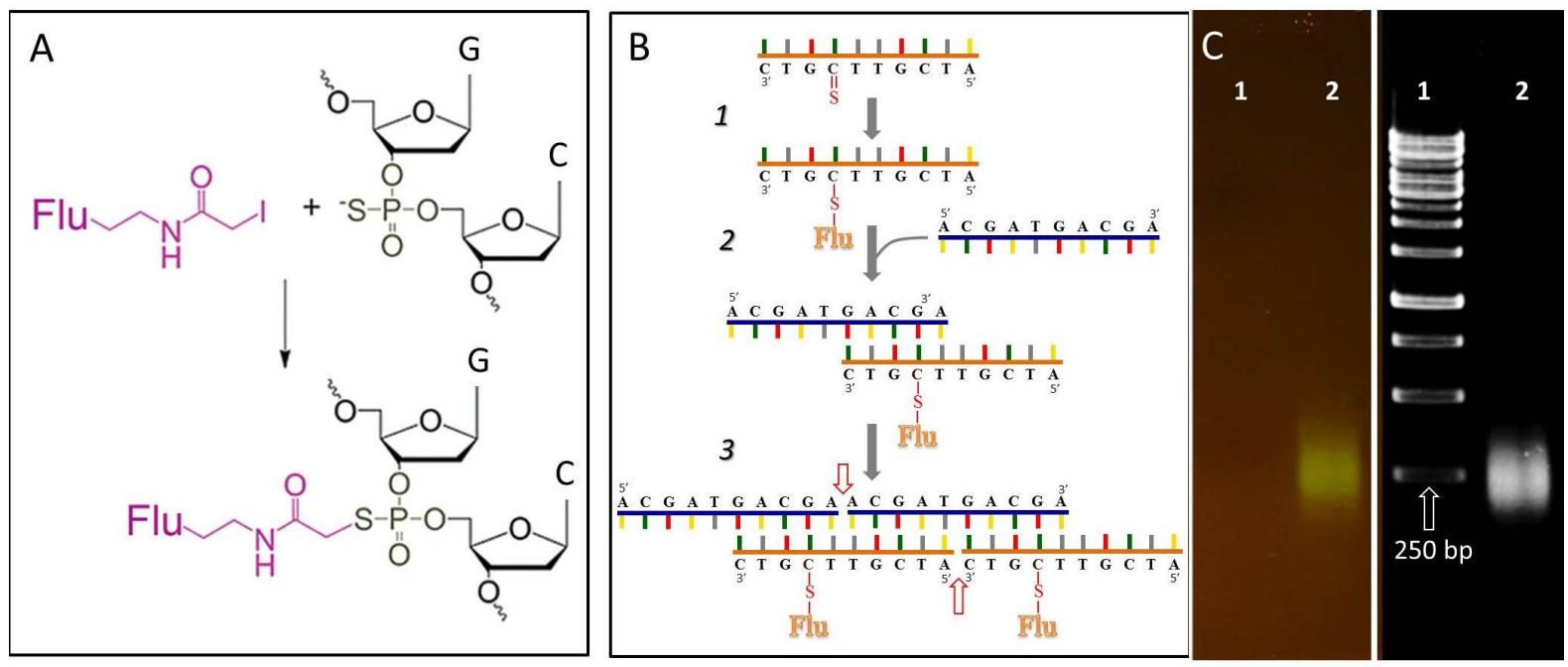

Figure 1. A. - Schematic illustration of conjugation of a phosphorothioate residue with 5-iodacetamidofluorescein (IAF). The dye interacts specifically with phosphorothioate residues; native nucleotides cannot bind IAF. B - The decameric oligonucleotide containing one phosphorothioated residue is being labeled with IAF (reaction 1). The labeled oligonucleotide forms a dimer with the half-complementary not-labeled one (reaction 2). The oligonucleotide dimers formed are being associated into long ds nucleic acid polymers in the presence of DNA ligase. The enzyme covalently links the gaps between the tandem oligonucleotide blocks in the strands (red arrows). C - Gel Electrophoresis of the synthesized Flu-labeled DNA concatemers (see B). The gel was illuminated by a blue light illuminator (at $470 \mathrm{~nm}$ ); the image was taken by a digital camera (left panel) or stained with ethidium bromide and imaged on a common gel imaging system (right panel). Lane 1 is a $1 \mathrm{~kb}$ DNA-ladder (the band corresponding to $250 \mathrm{bp}$ is indicated by the white arrow). 
one of the half-complementary nucleotides (see Fig.1A). We have conjugated the iodoacetamide derivative of fluorescein (5-iodacetamido-fluorescein) to the phosphorothioate residue of the decameric oligonucleotide as shown in Fig. 1A. The modified oligonucleotide was separated from the excess of the dye and purified to homogeneity using size-exclusion chromatography and ion-exchange HPLC. The purified oligonucleotide was incubated with an equimolar concentration of the half-complementary one in the presence of DNA ligase (see Fig. 1B). The synthesized molecules were purified by HPLC and analyzed by electrophoresis (see Fig. 1C). The resulting DNA molecules (about 250 bp) were highly fluorescent and can be visualized on the gel without staining with Ethidium Bromide (Fig. $1 \mathrm{C}$, left panel). By varying experimental conditions one can produced longer or shorter Fluorescein-labeled concatemeric molecules. We have also synthesized concatemers labeled with another fluorescent dye, 5-naphthalene-1-sulfonic acid (IAEDANS) and molecules containing both the above dyes.

The concatemers containing reactive groups along the whole length of the polymer can be used as templates for synthesis and assembly of novel materials and nanostructures. 